\title{
Measurement and analysis of electric potential decay in corona charged low-density polyethylene films
}

\author{
Z. Xu ${ }^{1 *}$, L. Zhang ${ }^{2}$ and G. Chen ${ }^{1}$ \\ ${ }^{1}$ School of Electronics and Computer Science, University of Southampton, \\ Southampton, SO17 1BJ, United Kingdom \\ ${ }^{2}$ Institute of Fluid Physics \\ China Academy of Engineering Physics \\ * E-mail: zx04r@ecs.soton.ac.uk
}

\begin{abstract}
In this paper, corona charged LDPE film was tested using a standard static potential monitor and the pulsed electro-acoustic (PEA) technique to observe the surface potential decay. A wide range of voltages and different corona charging times, different sample thicknesses and multi-layer films were used to demonstrate influential factors for surface potential decay. These provide a direct experimental evidence to support the bulk transport process. An important finding from the PEA results is that bipolar charge injection takes place during corona charging process and in subsequently decay process. The new evidence challenges the existing surface potential decay models which were developed based on a single charge carrier injection.
\end{abstract}

\section{INTRODUCTION}

In recent years there has been considerable interest in the surface potential decay characteristics of corona charged dielectrics. Various methods have been used to observe the decay including thermally stimulated discharge current, surface potential decay and the measurement of current/voltage during corona charging [1]. Different mechanisms have been proposed. Generally, there are three possible decaying routes for electric charge on surface, i.e. through the atmosphere, along the surface and transport through the bulk. In the present study, surface potential decay after corona charge deposition has been investigated for low density polyethylene (LDPE) films over a wide range of charging voltages and different charging times. To further understand decay process, experiments were carried out on multi-layer of LDPE films. In addition to monitor the surface potential, the pulsed electroacoustic (PEA) technique, which has been widely used to measure space charge in solid dielectrics, has been employed to measure charge distribution and its evolution in corona charged LDPE.

\section{EXPERIMENTAL DETAILS}

In order to reduce the influence of impurities, additivefree low density polyethylene film was selected. The thin planar films were purchased from the GoodFellow. Samples were cut into a disc shape with a diameter of $50 \mathrm{~mm}$, cleaned using methanol, raised in deionised water and dried by air.
LDPE film was charged in a conventional corona setup shown in Figure.1. The system consists of a high voltage needle, a wire mesh grid and a earth plate. The initial surface potential of corona charged film is controlled by the grid voltage applied across the needle and the rotatable earth electrode. After corona charging, the sample was moved to the static monitor (Compact JCI 140) quickly for the surface potential decay observation. The readings from the static monitor are proportional to the value of surface potential. After a calibration these readings can be easily converted into the surface potential. We use the negative corona charging in this study and the readings from the monitor were converted to absolute potential value in all the results. All experiments were carried out under a controlled environment where temperature and relative humidity were $21^{\circ} \mathrm{C}$ and $45 \%$, because both the temperature and relative humidity have an influence on charge decay.

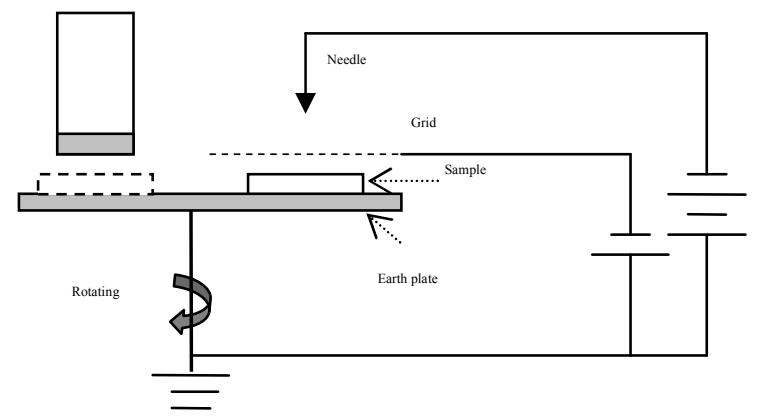

Figure 1 potential decay measurement system

The PEA technique is by far the most widely used method in space charge measurement. This technique utilizes the interaction between high voltage pulses and charge layers accumulated in the material to produce acoustic pressure waves. The pulsed acoustic waves correspond with each charge layer with respect to neutrality. The acoustic signals traverse across the material and are converted into an electrical signal by a piezo-electric transducer, amplified and captured with a digital oscilloscope. The detailed principle of PEA technique can be found in [2].After corona charging, both sides of sample were attached with a thin fresh LDPE film $(50 \mu \mathrm{m})$ very carefully to keep the deposited charges. The possible air bubble between films should be removed with small pressure because good contact 
between the films is necessary to get the good charge mapping results using the PEA method.

\section{RESULTS AND DISCUSSION}

The surface potential decay processes were measured for LDPE samples charged under different conditions initially. Charge distributions in the corona charged samples under the same charging condition were monitored by the PEA technique.

\section{Surface potential decay}

The potential decay result of corona-charged film $(50 \mu \mathrm{m})$ under different charging voltage for $2 \mathrm{mins}$ is shown in Figure 2. Surface potential shows an expected monotonic decay with time. However, we can observe clearly the crossover phenomenon; i.e. the surface potential in the sample with an initial high potential decays faster than that with a lower surface potential.

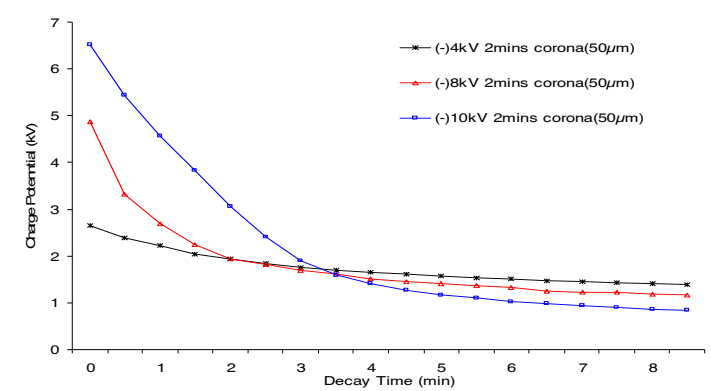

Figure 2: Potential decay under different corona voltage for thin sample $(50 \mu \mathrm{m})$

To explain the surface charge leakage and crossover phenomenon, different mechanisms have been proposed. The decay has been attributed to various mechanisms including recombination with opposite ions in air, surface migration and bulk conduction. The bulk process has been widely accepted and several models have been proposed to explain the surface potential decay [3-5]. To validate the bulk process responsible for surface charge decay, samples with different thicknesses $(180 \mu \mathrm{m}$ and $50 \mu \mathrm{m})$ were used under either the same corona voltage $(-8 \mathrm{kV})$ and or the same electric field $(\sim 80 \mathrm{kV} / \mathrm{mm})$. The decay results were shown in Figure 3 and Figure 4. The results in Figure 3 indicate that the material thickness plays a crucial role in surface potential decay. Both results in Figure 2 and Figure 3 show us that surface potential decay faster when the sample has an initial high electric field.

Figure 4 shows the potential decay under the same initial electric field for different thickness samples. To compare two curves, we moved down the higher corona voltage charged curve parallel. Overall, it can be seen that the decay rate for two samples has no significant difference except a little faster for the thinner sample at the beginning. From the above results, it is obvious that the surface potential decay strongly depends on the initial electric field of corona charging.

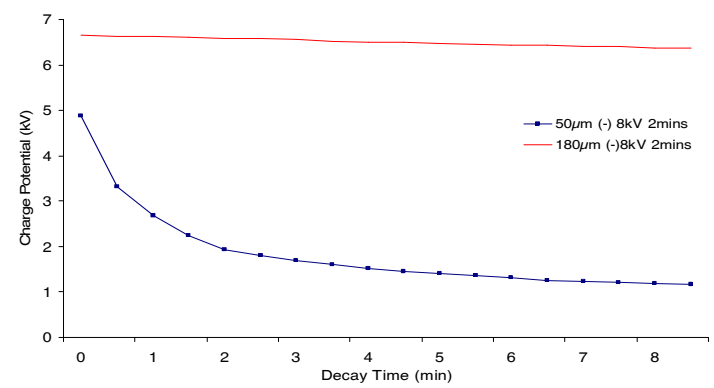

Figure 3: Potential decay under same corona voltage for different thickness sample.

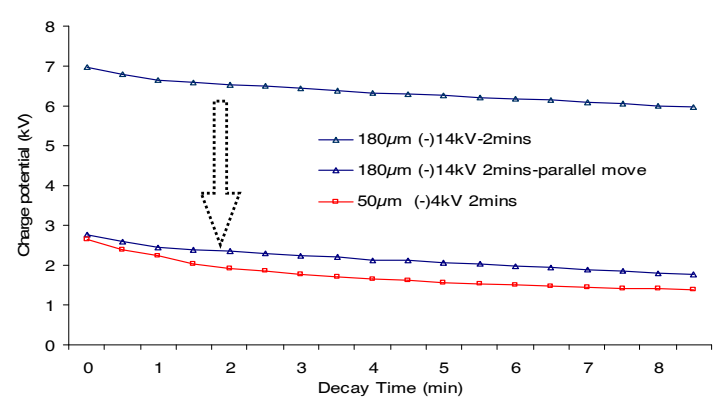

Figure 4: potential decay under same corona electric field.

Figure 5 shows the potential decay results of $50 \mu \mathrm{m}$ sample under $-8 \mathrm{kV}$ corona charging during different times. From this figure, it seems that the longer the charging time the faster the surface potential decays. This is especially true for first a few minutes. A possible reason for the faster decay is that the longer charging time allows more charge carriers injecting into the bulk. The injected charges then may move easily towards the opposite electrodes. This fast decay result has been validated by the PEA measurement data shown in the figure later.

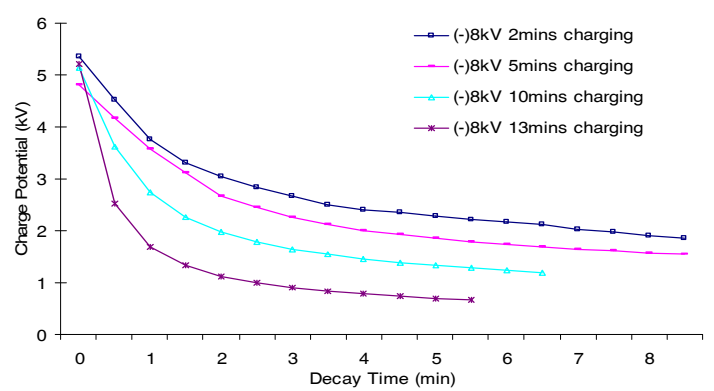

Figure 5: Potential decay during different charging period. 
Figure 6 shows the surface charge decay of multi-layer LDPE sample corona-charged under $-4 \mathrm{kV}$ for 2 mins. To achieve a similar electric field, the similar sample thickness was chosen; i.e. $180 \mu \mathrm{m}$ for one layer and three layers of $50 \mu \mathrm{m}(150 \mu \mathrm{m})$ as multi-layer. For the multi-layer potential decay monitoring, two measurements were carried out. In the first one the potential was continuously monitored. In the second measurement, after the potential was monitored for 3 minutes the top layer was carefully removed and surface potential produced by the middle and bottom layer was continuously observed and it shows a similar decay fashion. And after 5 minutes, the middle layer was removed and the potential produced by the bottom layer was measured as shown in Figure 6. Since surface potential is a representation of both surface charge and bulk charge, this result implies that electric charges exist either on the top surface of bottom two layers or in the bulk. No matter which case, the charges detected has to come from charge injection from the electrodes. Therefore, it provides a direct experimental evidence to support the bulk transport process.

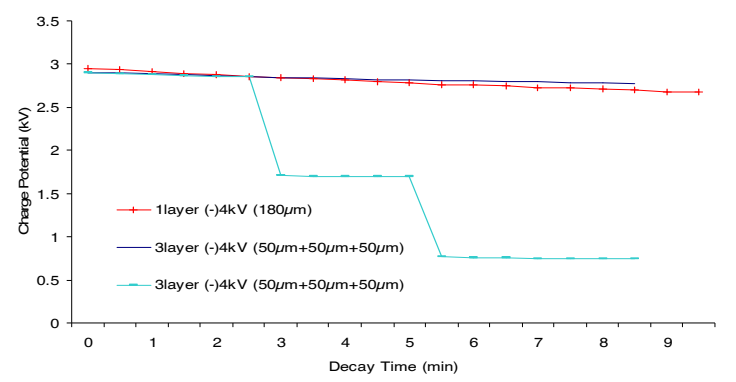

Figure 6: Potential decay for multi-layer sample $(-4 \mathrm{kV}$, $180 \mu \mathrm{m}, 50 \mu \mathrm{m}+50 \mu \mathrm{m}+50 \mu \mathrm{m})$

By comparing potential decay results for one layer and three layered samples, it can be noted that the surface potential from the multilayer sample with interface shows a slower decay than that from one layer sample. From the results it seems that the surface potential form a sample with an initial high field decays faster. Therefore, the surface potential from one layer sample $(180 \mu \mathrm{m})$ with an initial lower electric field should decay slower compared with the three layer sample $(150 \mu \mathrm{m})$. However, the results in Figure 6 contradict this. The only explanation lies in the presence of interfaces in the three layered sample. Interface seems to act as a barrier for charge movement therefore leading to a slow decay of surface potential.

\section{Charge mapping results}

It is clear that bulk process is responsible for the surface potential decay. As the surface potential changes relatively slow, it is possible to utilise the PEA technique to monitor space charge distribution and charge evolution.
From the results of surface potential measurement, we know that the decay rate is strongly depending on the experimental setting, e.g. corona voltage, sample thickness, applied electric field, charging period and polymeric interface. Although the potential decay can give us some information about the charge decay mechanism, more interesting thing is how the charges transport inside the sample during the decay period. Figure 7 shows the charge distribution of $180 \mu \mathrm{m}$ corona-charged under $8 \mathrm{kV}$ for 2 minutes. The two peaks at the PEA electrodes are induced charged peaks, which is definitely due to the existence of charge in the sample. The middle two peaks at top and bottom layer of corona charged sample are the charges formed during corona charging process, protected by the attached film. Negative charges presented at the top surface are expected as a process of charge deposition. However, a significant positive peak can be observed at the bottom surface of the sample. We believe this is because the charge injection from the ground electrode happened in the corona charged process. About the detailed discussion of bipolar injection can be found in our earlier works $[6,7]$. Charge evolution with time also shows a slow change, which is analogous to one of the potential decay curves shown in Figure 3.

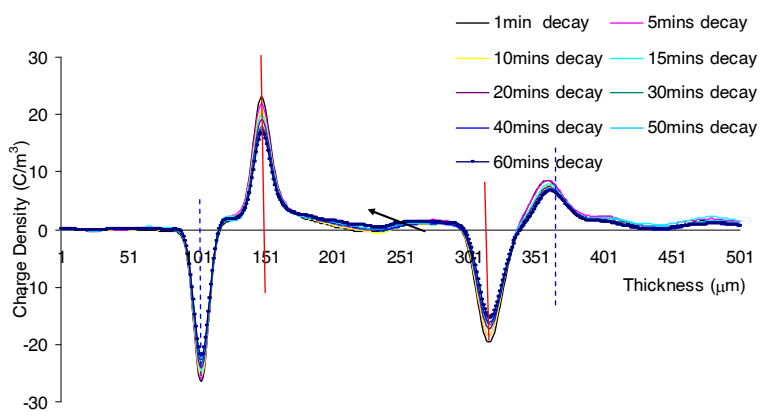

Figure 7: Space charge distribution in the corona charged sample $(180 \mu \mathrm{m},-8 \mathrm{kV} 2 \mathrm{mins}$ corona $)$

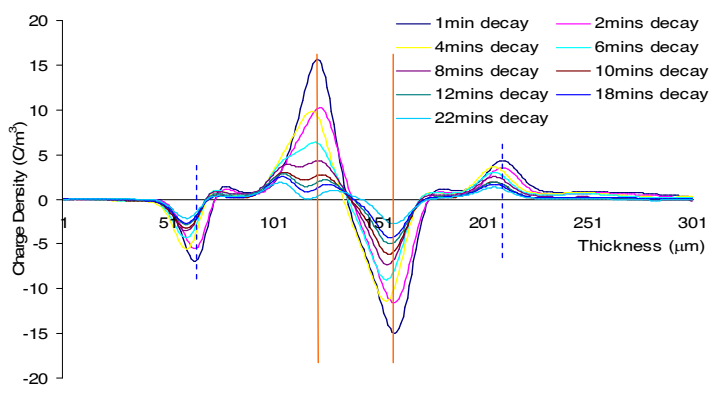

Figure 8: Space charge distribution in the corona charged sample $(50 \mu \mathrm{m},-8 \mathrm{kV}$ for $10 \mathrm{mins}$ corona charging)

Figure 8 shows the profile of space charge distribution decay in the corona-charged sample, under $-8 \mathrm{kV}$ for 10 minutes, the same experimental conditions as second bottom decay curves shown in Figure 5. It is noted from 
the PEA results that charges decrease extremely fast. This result is consistent with the result in Figure 5.

Figure 9 shows space charge distribution and dynamics in a two-layer LDPE sample. Compared with space charge distribution in one layer corona charged sample, an extra negative charge peak is observed at the interface between the two polymer layers. This can explain the early potential results obtained from the middle and bottom layer of the multi-layer sample (see Figure 6). These negative charges measured by the PEA or potential monitor are believed to be formed due to charge injection from corona charging surfaces and then transported to the interface. To study the charge transport in the bulk or at interface after corona charging, two-layer LDPE sample was charged for a long period and the PEA result is shown in Figure 10. There is a significant difference in charge distribution inside the bulk and interface of sample compared with a short charging period.

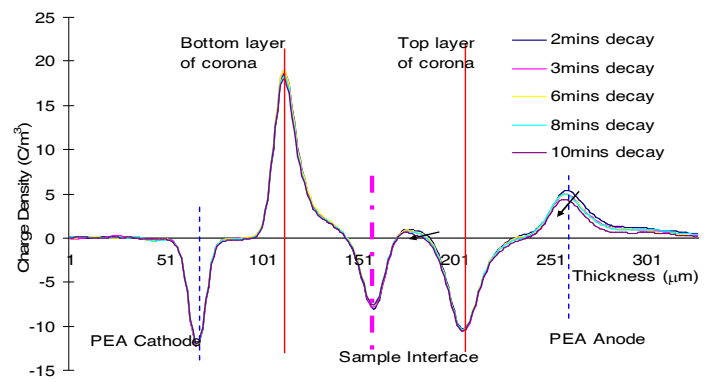

Figure 9: Space charge distribution decay in two layer corona charged sample $(50 \mu \mathrm{m}+50 \mu \mathrm{m},-4 \mathrm{kV} 2 \mathrm{mins}$ corona)

The negative peak at the top layer of corona charged sample is smaller than the peak shown in Figure 9 and deceased with time. Similar situation happens for the charge accumulated at the sample interface. An important result is negative charge dominated the bulk area of two corona charged layer. It seems that the negative charge deposited on the top layer moved into the bulk and overcome the interface barrier and transport in bulk of bottom layer. The longer the corona charging time the more electrons injected into the bulk. From the charge distribution evolution, it is easy to know that charge decays faster in the sample corona charged for a long time than that in the sample coronacharged for a short period. This is consistent with the results shown in Figure 5.

Using the charge mapping technique, the bipolar charge carrier injection has been observed. These new phenomenon challenges the existing surface potential decay models, which were established based on the single layer of charge carries. And interface influence factor should be considered in further research on corona charge polymeric materials as well.

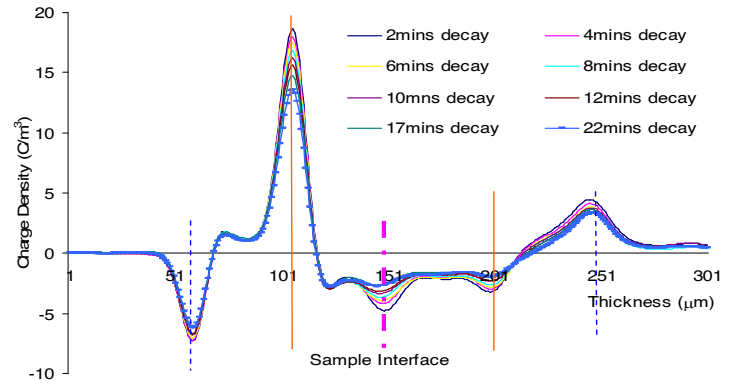

Figure 10: Space charge distribution decay in two layer corona charged sample $(50 \mu \mathrm{m}+50 \mu \mathrm{m},-4 \mathrm{kV}$ for $20 \mathrm{mins})$

\section{CONCLUSION}

The crossover phenomenon has been experimentally confirmed in this study. The surface potential decay is determined by several factors: corona voltage, sample thickness, charging electric field, charging period, polymer interface. The charge mapping technique used in the study of surface potential decay in polyethylene films is a valuable attempt. This technique provides an alternative way to investigate charge decay process and it allows monitoring charge migration through the bulk of corona charged film. The charge profiles obtained strongly suggest that the bipolar charge injection has taken place and interface has a special influence on charge transfer through the sample. And new surface potential decay model is required to explain the charge transport processes in corona charged LDPE film.

\section{REFERENCES}

[1] A. Kumadaa, Y. Shimizub, M. Chibab and K. Hidakab, Pockels Surface Potential Probe and Surface Charge Density Measurement, Journal of Electrostatics vol. 58, p. 45-58, 2003.

[2] G. Chen, Y. L. Chong and M. Fu, Calibration of the Pulsed Electroacoustic Technique in the Presence of Trapped Charge, Measurement Science and Technology vol. 17, p. 974-1980, 2006.

[3] T. J. Sonnonstine and M. M. Perlman, SurfacePotential Decay in Insulators with Fielddependent Mobility and Injection Efficiency, J. App. Phys. vol. 46, p. 3975 - 3981, 1975.

[4] R. Toomer, T. J. Lewis, Charge Trapping in CoronaCharged Polyethylene Films, J. Phys. D: Appl. Phys. vol. 13, p. 1343-1356, 1980.

[5] H. v. Berlepsch, Interpretation of Surface Potential Kinetics in HDPE by a Trapping Model, J.Phys. D:Appl. Phys. Vol. 18, p. 1155 - 1170, 1985.

[6] G. Chen, Z. Xu and L. W. Zhang, Measurement of Surface Potential Decay of Corona-Charged Polymer Films Using the Pulsed Electroacoustic Method, Measurement Science and Technology, 2007.

[7] L. W. Zhang, Z. Xu and G. Chen, Charge Decay of Corona-Charged Low Density Polyethylene, Electrostatics 2007, Oxford, UK, 2007. 